\title{
Phenylketonuria: No Specific Frontal Lobe-Dependent Neuropsychological Deficits of Early-Treated Patients in Comparison with Diabetics
}

\author{
REINHOLD FELDMANN, JONAS DENECKE, MICHAEL PIETSCH, \\ MICHAEL GRENZEBACH, AND JOSEF WEGLAGE
}

Department of Pediatrics, University of Münster, D-48129 Münster, Germany

\begin{abstract}
ABST
Neuropsychologic studies have shown that even phenylketo-
nuric patients treated early suffer from phenylalanine-related
deficits in all age periods, from childhood to adulthood. This
study was performed to determine whether phenylketonuric chil-
dren show specific frontal lobe-dependent deficits when com-
pared with diabetic patients. The comparative study included 42
phenylketonuric patients, 10 to 18 y of age [mean 14.7 (years,
months), SD 2.9], and 42 diabetic patients matched for sex, age,
and socioeconomic status. Patients were assessed for intelligence
quotient (Culture Fair Intelligence Test), information processing
(Wisconsin Card Sorting Test, Trail-Making Test), and selective
(Stroop task) as well as sustained attention (Test d-2). Phenylk-
etonuric patients had significantly poorer results than the diabetic
patients. Within all tests, however, this was due to reduced
performance speed, not to deficits in specific functions. Patients
did not show deficits in insight and learning. The selection
abilities and the sustained attention of the phenylketonuric pa-
\end{abstract}
When patients with PKU are restricted to a diet low in PHE soon after birth, their neurologic and intellectual development is close to normal (1). Duration and strictness of the diet are still under discussion (2). Until recently, the policy was to relax the diet after $10 \mathrm{y}$ of age, however, current recommendations suggest maintaining this strict diet for as long as possible $(2,3)$.

PKU resulting from PHE hydroxylase deficiency [McKusick 26160 (4)] is the most frequent inborn error of amino acid metabolism (5). Longitudinal studies have shown that the brain is most vulnerable to elevated PHE levels during the first years of life. After the age of 8 to $10 \mathrm{y}$, no independent influence of blood PHE levels on measures of general ability (intelligence) could be demonstrated $(1,6-8)$. Nevertheless, in all age

Received November 9, 2000; accepted August 21, 2001.

Correspondence: Dr. Reinhold Feldmann, Department of Pediatrics, University of Münster, Albert-Schweitzer-Str. 33, D-48129 Münster, Germany; e-mail: feldrei@uni-muenster.de

Supported, in part, by the SHS Company, Heilbronn, Germany.

DOI: 10.1023/01.PDR.0000017479.64723.30 tients were not impaired. Performance speed and blood phenylalanine levels were negatively correlated. Elevated phenylalanine levels may cause an imbalance in neurotransmitter metabolism. However, this seems to refer to a global neurotoxic effect rather than to specific effects on the dopaminergic system, which would affect specifically the activation of the frontal lobes. (Pediatr Res 51: 761-765, 2002)
Abbreviations:
CFT 20, Culture Fair Intelligence Test-Scale 2
CWIT, Color-Word-Interference-Task
HBA1, glycohemoglobin $A_{1}$
PHE, phenylalanine
PKU, phenylketonuria
WCST, Wisconsin Card Sorting Test
ZVT, German Number Combination Test
(Zahlen-Verbindungs-Test)

periods (childhood to adulthood), neuropsychological deficits were reported for PKU patients (9-15). A review of 21 published studies reported simple motor speed, speech, language, memory, and basic logic to be generally unaffected (16). Deficits were primarily found in abstract reasoning, executive functioning, and the attention area of information processing (16).

Elevated PHE levels can interfere with the development and function of the CNS by different mechanisms, such as dysmyelination (17) and decrease of neurotransmitter receptor density (18). Besides these morphologic changes, elevated PHE levels may cause an imbalance in neurotransmitter metabolism (1922). PHE inhibits the uptake of the precursor amino acids tyrosine and tryptophan into the brain as well as the synthesis of the neurotransmitters dopamine and serotonin and possibly their neuronal release (19-22). In contrast to the serotonin projections, the dopaminergic system in the neocortex is highly topographically organized (23). The mesolimbic and mesocortical systems project from the ventral tegmentum to limbic and 
cortical areas, especially to the anterior cingulate and the frontal lobes $(21,24)$. Weglage et al. (25) found deficits in selective and sustained attention processes in early-treated children with PKU compared with healthy controls. These deficits may reflect specific impairments of frontal lobe functions in patients with PKU, reported by Welsh et al. (26). Authors postulated specific neuropsychologic deficits of frontal lobe functions resulting from PHE-related central dopamine deficiency (27).

However, it is still unknown whether the reported deficits in selective and sustained attention processes are specific to PKU if compared with another chronic illness rather than to healthy controls. We therefore investigated whether information processing as well as selective and sustained attention processes in children with PKU treated early show a specific profile if compared with patients with diabetes mellitus type I. Diabetic patients as controls were chosen for several reasons. Diabetics and PKU patients suffer from chronic metabolic illnesses that are comparably burdensome (27). At the same time, both groups of patients do not differ significantly either in the degree or in the pattern of their psychosocial profile measured using the Child Behavior Checklist (27). Psychosocial maladjustment that may affect cognitive functioning is controlled better this way than using healthy controls. Finally, no illness-related impairment of frontal lobe functioning may be expected in adolescent diabetic patients if hypoglycemia is excluded. The study was approved by the applicable institutional review board and informed consent was obtained from all patients.

\section{PATIENTS AND METHODS}

We examined 42 early-treated patients (start of diet before wk 7 of life; mean 18 d, SD 9 d) with classical PKU (19 females, 23 males), 10 to $18 \mathrm{y}$ of age (mean $14.7 \mathrm{y}$, SD $2.9 \mathrm{y}$ ) and 42 patients with diabetes mellitus type I (manifestation of diabetes 2-14 y earlier, mean 7.4 y, SD 4.2 y) matched for age (10-18 y, mean 14.8 y, SD 2.7 y), sex (19 females, 23 males), and socioeconomic status. Based on the father's educational background and occupation, the families were classified according to the following five categories of socioeconomic status: upper (PKU 1/diabetics 1), upper-middle (PKU 3/diabetics 3), middle (PKU 23/diabetics 24), lower-middle (PKU 10/diabetics 9), and lower (PKU 5/diabetics 5). The school achievement was evaluated from parents' ratings with respect to the following variables:

1. Age at start of schooling

2. Number of patients who had to repeat at least one class

3. Types of schools attended

Blood levels of PHE and HBA1 were determined by HPLC methods following standard procedures. Lifelong metabolic control was estimated by calculating the mean of all yearly medians of PHE and HBA1 levels since start of treatment. In addition, the concurrent PHE and HBA1 levels at the time of investigation were determined.

Patients were assessed for intelligence quotient (IQ) (CFT 20), information processing (WCST, Trail-Making Test), and selective (Stroop task) and sustained attention (Test d-2). The test battery administered in addition to the intelligence test was limited to challenging instruments that have previously demonstrated their appropriateness in detecting deficits in frontal lobe function.

Intelligence was measured by the CFT 20. The test was developed and standardized by Weiss (28) in 1987. It is a "culture-fair" IQ test, which does not depend on language skills. Internal consistency of the test ranged from $r=0.90$ to 0.95 , and test-retest correlations ranged between $r=0.90$ and 0.97 (reported from the CFT 20 manual).

Information processing was measured by the WCST, a neuropsychological task that requires subjects to categorize geometric forms by their color, number, or shape. To be successful on the WCST, subjects need to attain and then maintain that concept, but switch it when the rules for sorting change. This task is difficult for patients with frontal lesions $(29,30)$. Studies showed that better performance in the WCST correlated with greater metabolic activation in the dorsolateral prefrontal region (31). WCST scores are calculated from the number of wrong matches, the number of categories completed, and the trials needed to complete the first category. Additional assessments consider the failure to maintain sets of correct matching and the learning abilities of subjects. Here, scores are calculated by counting incomplete categories and computing mean number of trials to complete categories, respectively.

Trail-making tests are used widely in the screening of psychomotor function. They require visual-motor tracking and basic sequencing skills. Trail changes require that the subjects shift between rehearsed sequences, thus providing a measure of sequencing skills. The ZVT was standardized by Oswald and Roth (32) in 1987. The ZVT consists of four subtests in which different trails are to be made. The mean time needed to make the trails is calculated as a score of information processing speed.

The CWIT according to Stroop consists of three parts: reading of color words, color naming, and the interference task. Subjects note the strong interference of word reading with color naming, called the Stroop interference effect (e.g. the word "red" printed in green requires the verbal response "green"). Additionally, a nomination score is quantitated in terms of the difference in reaction times of reading of color words and color naming. The tendency to interference (selection) is quantitated in terms of the difference in reaction times of color naming and the interference task. The German adaptation of the Stroop task was standardized by Bäumler (33) in 1984. Evaluation of results was done on the basis of raw data (in seconds) because the small normative sample of the test was not representative for sex, age, and education. The German adaptation of the Stroop task is comparable to the test versions used in positron emission tomography studies by Pardo et al. (34) and Bench et al. (35), who demonstrated an activation of the frontal lobes during the Stroop test in healthy subjects. Using a similar version of the Stroop task, Perret (36) reported selective deficits of frontal damaged patients on this test.

Sustained attention was assessed by the Test $\mathrm{d}-2$, a paper and pencil test that measures speed and accuracy in differentiating similar visual stimuli. The test was revised in 1994 (37). Internal consistency of the test ranged from $r=0.84$ to 0.98 , 
Table 1. Serum PHE concentrations: two indices of dietary control $(\mu \mathrm{mol} / \mathrm{L})$

\begin{tabular}{ll}
\hline & Mean \pm SD (Range) \\
\hline $\begin{array}{l}\text { Serum PHE level at the time of testing }(n=40) \\
\text { Mean of yearly medians of serum PHE levels } \\
\text { throughout life }(n=35)\end{array}$ & $526 \pm 412(72-1746)$ \\
\end{tabular}

test-retest correlations ranged from $r=0.88$ to 0.92 (reported from the test manual). Results are expressed in the following scores: $\mathrm{N}$ (number of items completed) and $\mathrm{M}$ (mistakes, i.e. missed target items plus commissions). $\mathrm{N}$ is described as a "measure of basic cognitive working speed," and $\mathrm{M}$ is described as an index of "selection abilities" (37).

Within the results, the mean test scores reached by the phenylketonuric and diabetic patients are reported with reference to the mean scores reached by the standardization populations of the tests used. These scores can be seen as normal scores of healthy subjects.

Data analysis was based on $t$ tests for independent samples and on Pearson correlations. The significance tests were of an explorative nature; the level of significance was set at $p<0.01$. Statistical analyses were carried out using SPSS 9.0 (SPSS Inc., Chicago, IL, U.S.A.).

\section{RESULTS}

In general, patients followed a diet recommended by the German Pediatric Society [ $240 \mu \mathrm{mol} / \mathrm{L}$ up to the age of $10 \mathrm{y}$, $900 \mu \mathrm{mol} / \mathrm{L}$ up to the age of $15 \mathrm{y} \mathrm{(38)]} \mathrm{(Table} \mathrm{1).}$

The 42 patients with PKU reached an IQ of 99.58 (SD 13.68, range 76-127), which is not significantly different from the mean IQ of the normal population (100, SD 15). This is true for the IQ of the diabetic patients (104.38, SD 12.16, range 79-132). There were no statistically significant IQ differences between groups of patients.

Information processing measured by the WCST did not show significant differences between groups. Patients with PKU needed more trials to complete the first category. Having attained a concept, however, they performed as well as the diabetic patients. Patients with PKU showed moderately better learning abilities and completed as many categories as the diabetic patients. In general, both groups met the scores of the normative, healthy sample (Table 2).

Information processing measured by the ZVT showed significant differences between groups. The mean scores reached by the diabetic patients met the mean scores of the ZVT standardization population, whereas patients with PKU needed considerably more time to make the trails (Table 3 ).

Using the Test $\mathrm{d}-2$, the PKU patients reached a performance level significantly below that of the diabetic patients (Table 4). However, their low performance results from a reduced number of items completed, not an increased number of mistakes. The scores of the diabetics were similar to the mean scores of the Test $\mathrm{d}-2$ standardization population.

In most of the CWIT subtests, the PKU patients had significantly poorer results than the diabetic patients (Table 5). They showed reduced speed in reading and naming as well as in the interference task. Here, expected nomination speed is reduced but selection is not.

Negative Pearson correlations of blood PHE levels with test scores were significant for processing speed measured by the ZVT only. A significant positive correlation was found between serum PHE levels of the patients with PKU and the number of mistakes made in the Test d-2 (Table 6).

\section{DISCUSSION}

Neuropsychologic studies of PKU revealed deficits primarily found in abstract reasoning, executive functioning, and the attention area of information processing. Children of all ages were equally likely to show problem-solving deficits and slower reaction times, and performances were more closely related to the concurrent PHE level than to long-term dietary status $(10,11,13,16,25,26)$. Recently, Welsh et al. (26) and Diamond (11) postulated specific neuropsychologic deficits of frontal lobe functions due to PHE-related central dopamine deficiency. This seemed to be plausible because the dopaminergic system of the brain is highly topographically organized, including projections to the frontal lobes (23). In addition, reduced central concentrations of dopamine and metabolites of dopamine in cases of elevated PHE levels were demonstrated (21). Waisbren et al. (16), in their review of 21 neuropsychologic studies in PKU, concluded that "samples available for study were generally small and heterogeneous with regards to age, treatment history, current dietary status and intellectual capabilities." Until now, the question of whether PKU patients suffer from specific deficits of frontal lobe functions remained to be answered.

In our study, patients with PKU showed significantly poorer performance in information-processing speed. They needed considerably more time to complete trails of the ZVT and needed more trials to complete the first category of the WCST.

Table 2. Results of the WCST in children with PKU and diabetes

\begin{tabular}{lccc}
\hline \multicolumn{1}{c}{ Mean \pm SD (Range) } & PKU Patients & Diabetic Patients \\
$(n=42)$ & $18 \pm 9(6-44)$ & NS \\
Total number of errors & $21 \pm 13(0-51)$ & $3.1 \pm 1.3(0-5)$ & NS \\
Number of categories completed & $3.3 \pm 2.2(0-13)$ & $12 \pm 3(0-23)$ & $<0.01$ \\
Trials to complete first category & $15 \pm 11(3-64)$ & $0.4 \pm 0.5(0-2)$ & NS \\
Failure to maintain set & $0.3 \pm 0.5(0-2)$ & $-11 \pm 20(-52-46)$ & NS \\
Learning to learn & $-8 \pm 28(-77-55)$ & & \\
\hline
\end{tabular}

According to the WCST manual, mean numbers of the normal population are as follows: errors, 17; categories completed, 3-6; trials to complete first category, $10-13$; failure to maintain set, $0-2$; learning to learn, $\geq-6.99$.

A $p$ value $>0.01$ is NS. 
Table 3. Results of the Trail-Making test in children with PKU and diabetes

\begin{tabular}{lccc}
\hline Mean $\pm \mathrm{SD}$ (Range) & $\begin{array}{c}\text { PKU Patients } \\
(n=42)\end{array}$ & $\begin{array}{c}\text { Diabetic Patients } \\
(n=42)\end{array}$ & $p$ Value \\
\hline T value & $41 \pm 10(20-64)$ & $50 \pm 12(28-75)$ & $<0.001$ \\
Time to complete trail(s) & $105 \pm 31(64-204)$ & $82 \pm 25(44-154)$ & $<0.001$ \\
\hline
\end{tabular}

Mean $\mathrm{T}$ value (time) of the normal population: $50 \mathrm{~s}, \mathrm{SD}=10 \mathrm{~s}$.

Table 4. Results of the Test $d-2$ in children with PKU and diabetes

\begin{tabular}{lccc}
\hline Mean \pm SD (Range) & $\begin{array}{c}\text { PKU Patients } \\
(n=42)\end{array}$ & $\begin{array}{c}\text { Diabetic Patients } \\
(n=42)\end{array}$ & $p$ Value \\
\hline $\mathrm{N}$ (percentile) & $31 \pm 28(0-99)$ & $45 \pm 32(1-99)$ & $<0.01$ \\
M (percentage) & $10 \pm 17(1-77)$ & $6 \pm 7(0-30)$ & NS \\
\hline
\end{tabular}

$\mathrm{N}$, number of items completed; $\mathrm{M}$, number of mistakes. Mean percentile of the normal population $=50$.

A $p$ value $>0.01$ is NS.

Table 5. Results of the CWIT in children with PKU and diabetes

\begin{tabular}{lcll}
\hline $\begin{array}{c}\text { Mean } \pm \text { SD } \\
\text { (Range) }\end{array}$ & $\begin{array}{c}\text { PKU Patients } \\
(n=42)\end{array}$ & $\begin{array}{c}\text { Diabetic Patients } \\
(n=42)\end{array}$ & $p$ Value \\
\hline CWR & $42 \pm 19(24-129)$ & $34 \pm 7(22-61)$ & $<0.01$ \\
CN & $70 \pm 17(44-112)$ & $56 \pm 13(37-85)$ & $<0.001$ \\
INT & $116 \pm 36(41-185)$ & $89 \pm 28(38-150)$ & $<0.001$ \\
NOM & $42 \pm 8(26-62)$ & $48 \pm 11(29-71)$ & $<0.01$ \\
SEL & $56 \pm 10(30-74)$ & $58 \pm 11(36-80)$ & NS \\
\hline
\end{tabular}

CWR, color word reading; CN, color naming; INT, interference task; NOM, expected nomination speed; SEL, selection; all scores in seconds.

A $p$ value $>0.01$ is NS.

However, PKU patients managed to obtain a number of completed categories that did not differ from the performance of diabetic patients or healthy controls. According to the WCST learning-to-learn score, the learning abilities of patients with PKU are not impaired. PKU patients showed poorer performance in reaction speed but not in selective attention. Patients with PKU did complete significantly less items of the Test d-2, which is indicative of a low cognitive working speed. However, they did not show impaired selection abilities, as far as mistakes made in the Test d-2 are concerned. Patients with PKU showed an increased reaction time in reading of color words and color naming as well as in the interference task. Here, expected nomination speed was reduced, but selection (the difference in reaction times of color naming and the interference task) was not.

The pattern of differences between PKU and diabetic patients matched for age, sex, and socioeconomic status demonstrated PHE-related reduced performance speed but no specific deficits in frontal lobe functions such as selection abilities, insight, or learning. In general, our results again have demonstrated the influence of concurrent PHE levels on neuropsychologic test results. Even in adolescence and early adulthood, elevated PHE levels are associated with cognitive impairment primarily in terms of prolonged reaction times. This is in accordance with recent studies of Burgard et al. (10) and Griffiths et al. (13).

As far as adolescents and young adults are concerned, our results do not support the hypothesis of Welsh et al. (26) and Diamond (11) postulating a specific frontal lobe deficiency in PKU due to a PHE-related central dopamine deficiency. Recently, Ullrich et al. (39), who treated adult PKU patients with L-dopa, was not able to demonstrate any significant effect of drug therapy on neuropsychological test results depending on frontal lobe functions. They concluded that there may be no functionally relevant dopamine deficiency in PKU patients.

Our results are also in accordance with findings of Pietz et al. (40). Using magnetic resonance spectroscopy for quantitative measurement of PHE levels in brain of PKU patients, they found no specific or localized pathology in the EEG, but a general slowing of background activity caused by elevated brain PHE levels after an oral PHE load. After blocking PHE uptake into the brain by supplementing high doses of other large neutral amino acids, there was no slowing of EEG activity (40).

Table 6. Pearson correlations of serum PHE levels with test scores

\begin{tabular}{lcc}
\hline & $\begin{array}{c}\text { Mean of Yearly Medians of } \\
\text { Serum PHE Levels } \\
\text { throughout Life }(n=35)\end{array}$ & $\begin{array}{c}\text { Serum PHE Level at } \\
\text { the Time of Testing } \\
(n=40)\end{array}$ \\
\hline IQ (CFT 20) & -.108 & -.182 \\
Total number of errors (WCST) & -.256 & -.144 \\
Number of categories completed (WCST) & -.198 & -.155 \\
Trials to complete first category (WCST) & .007 & -.257 \\
Failure to maintain set (WCST) & -.146 & -.232 \\
Learning to learn (WCST) & .033 & -.146 \\
T value (ZVT) & .040 & .246 \\
Sec (ZVT) & $-.430^{*}$ & $-.520 \dagger$ \\
N (d-2) & .329 & .126 \\
M (d-2) & $.356 *$ & .208 \\
N - M (d-2) & .068 & -.023 \\
CWR (CWIT) & .282 & -.111 \\
CN (CWIT) & .229 & .033 \\
INT (CWIT) & .185 & .028 \\
NOM (CWIT) & -.295 & .003 \\
SEL (CWIT) & .129 & .055 \\
\hline
\end{tabular}

$\mathrm{Sec}$, time needed to complete trail; N, number of items completed; M, mistakes; $\mathrm{N}-\mathrm{M}$, number of items completed minus mistakes; CWR, color word reading; CN, color naming; INT, interference task; NOM, expected nomination speed; SEL, selection.

$* p<0.01 ; \dagger p<0.001$. 
In summary, it seems to be more appropriate to characterize patients with early-treated PKU as performing qualitatively normally on a reduced speed level than to perform qualitatively differently from diabetic patients and healthy controls. Further studies will have to clarify the specific pathophysiological mechanisms of this general cognitive slowing as a consequence of elevated PHE levels in PKU. In addition, there remains the determination of the practical relevance of neuropsychologic effects resulting from elevated PHE levels.

Acknowledgment. The authors thank the patients and their parents for participating in this study.

\section{REFERENCES}

1. Smith I, Beaseley MG, Ades AE 1991 Effect on intelligence of relaxing the low phenylalanine diet in phenylketonuria. Arch Dis Child 65:311-316

2. Milner JD, Wurtman RJ 1986 Effects of phenylalanine on the release of endogenous dopamine from rat striatal slices. J Neurochem 47:1444-1448

3. Smith I 1994 Treatment of phenylalanine hydroxylase deficiency. Acta Paediatr Suppl 407:60-65

4. McKusick VA, Francomano CA, Antonarakis SE, Pearson PL 1994 Mendelian Inheritance in Man. A Catalog of Human Genes and Genetic Disorders, 11th Ed. The Johns Hopkins University Press, Baltimore, pp 2113-2124

5. Scriver CR, Kaufman S, Eisensmith RC, Woo SLC 1995 The hyperphenylalaninemias. In: Scriver CR (ed) Inherited Disease, Vol 1. McGraw-Hill, New York, pp $1015-1075$

6. Burgard P, Schmidt E, Rupp A, Schneider W, Bremer HJ 1996 Intellectual development of the patients of the German Collaborative Study of patients treated for phenylketonuria. Eur J Pediatr 155(suppl 1):S33-S38

7. Holtzman NA, Kronmal RA, van Doorninck W, Azen C, Koch R 1986 Effect of age at loss of dietary control on intellectual performance and behavior of children with phenylketonuria. N Engl J Med 314:593-598

8. Rey F, Abadie V, Plainguet F, Rey J 1996 Long-term follow up of patients with classical phenylketonuria after diet relaxation at 5 years of age. The Paris Study. Eur J Pediatr 155(suppl 1):S39-S44

9. Brunner RL, Berry HK 1987 Phenylketonuria and sustained attention: the continuous performance test. Int J Clin Neuropsychol 9:68-70

10. Burgard P, Rey F, Rupp A, Abadie V, Rey J 1997 Neuropsychological functions in early treated patients with phenylketonuria on and off diet. Results of a cross-national and cross-sectional study. Pediatr Res 41:368-347

11. Diamond A 1994 Phenylalanine levels of 6 to $10 \mathrm{mg} / \mathrm{dl}$ may not be as benign as once thought. Acta Paediatr Suppl 407:89-91

12. Faust D, Libon D, Pueschel S 1986 Neuropsychological functioning in treated phenylketonuria. Int J Psychiatr Med 16:169-177

13. Griffiths P, Campbell R, Robinson P 1998 Executive function in treated phenylketonuria as measured by the one-back and two-back versions of the continuous performance task. J Inherit Metab Dis 21:125-135

14. Schmidt E, Burgard P, Rupp A 1996 Effects of concurrent phenylalanine levels on sustained attention and calculation speed in patients treated early for phenylketonuria. Eur J Pediatr 155(suppl 1):82-88

15. Schmidt E, Rupp A, Burgard P, Pietz J, Weglage J, Sonneville de L 1994 Sustained attention in adult phenylketonuria: the influence of the concurrent phenylalanine blood level. J Clin Exp Neuropsychol 16:681-688

16. Waisbren SE, Brown MJ, Sonneville de LMJ, Levy HL 1994 Review of neuropsychological functioning in treated phenylketonuria: an information processing approach. Acta Paediatr Suppl 407:98-103
17. Cleary MA, Walter JH, Wraith JE, Jenkins SM, Alani SM, Tyler K, Whittle D 1994 Magnetic resonance imaging of the brain in phenylketonuria. Lancet 344:87-90

18. Hommes FA 1994 Loss of neurotransmitter receptors by hyperphenylalaninemia in the HPH-5 mouse brain. Acta Paediatr Suppl 407:120-121

19. Lou HC, Güttler F, Lykkelund C, Bruhn P, Niederwieser A 1985 Decreased vigilance and neurotransmitter synthesis after discontinuation of dietary treatment for phenylketonuria in adolescents. Eur J Pediatr 144:17-20

20. Lou HC, Lykkelund C, Gehrdes AM, Udesen H, Bruhn P 1987 Increased vigilance and dopamine synthesis by large doses of tyrosine or phenylalanine restriction in phenylketonuria. Acta Paediatr 76:560-565

21. McKean CM 1972 The effects of high phenylalanine concentrations on serotonin and catecholamine metabolism in the human brain. Brain Res 47:469-476

22. Pratt OE 1992 Transport inhibition in the pathology of phenylketonuria and other inherited metabolic diseases. J Inherit Metab Dis 2(suppl 5):75-81

23. Divac I, Bjorklund A, Lindvall O, Passingham R 1978 Converging projections from the mediodorsal thalamic nucleus and mesencephalic dopaminergic neurons to the neocortex in three species. J Comp Neurol 180:59-72

24. Brass CA, Greengard O 1982 Modulation of cerebral catecholamine concentrations during hyperphenylalaninemia. Biochem J 208:765-771

25. Weglage J, Pietsch M, Fünders B, Koch HG, Ullrich K 1996 Deficits in selective and sustained attention processes in early treated children with phenylketonuria —result of impaired frontal lobe functions? Eur J Pediatr 155:200-204

26. Welsh MC, Pennington BF, Ozonoff S, Rouse B, McCabe ERB 1990 Neuropsychology of early-treated phenylketonuria: specific executive function deficits. Child Dev 61:1697-1713

27. Weglage J, Grenzebach M, Pietsch M, Feldmann R, Linnenbank R, Denecke J, Koch HG 2000 Behavioral and emotional problems in early treated adolescents with phenylketonuria in comparison to diabetic patients and healthy controls. J Inherit Metab Dis 23:487-496

28. Weiss RH 1987 Grundintelligenztest Skala 2 CFT 20. Hogrefe, Göttingen, pp 42-45

29. Milner B 1963 Effects of different brain lesions on card sorting. Arch Neurol 9:90-100

30. Robinson AL, Heaton RK, Lehman AW, Stilson DW 1980 The utility of the Wisconsin Card Sorting Test in detecting and localizing frontal lobe lesions. J Consult Clin Psychol 48:605-614

31. Weinberger DR, Berman KF, Zec RZ 1986 Physiologic dysfunction of dorsolateral prefrontal cortex in schizophrenia. I: regional cerebral blood flow evidence. Arch Gen Psychiatry 43:114-124

32. Oswald DW, Roth E 1987 Der Zahlen-Verbindungs-Test (ZVT). 2. Auflage. Hogrefe, Göttingen, pp 15-19

33. Bäumler G 1985 Farbe-Wort-Interferenztest(FWIT)nach JR Stroop. Hogrefe, Göttingen, pp $10-14$

34. Pardo JV, Pardo PJ, Janer KW, Raichle ME 1990 The anterior cingulate cortex mediates processing selection in the Stroop attentional conflict paradigm. Proc Natl Acad Sci USA 87:256-259

35. Bench CJ, Frith CJ, Grasby PM, Friston KJ, Frackowiak RSJ, Dolan RJ 1993 Investigations of the functional anatomy of attention using the Stroop test. Neuropsychologia 31:907-922

36. Perret E 1974 The left frontal lobe of man and the suppression of habitual responses in verbal categorical behaviour. Neuropsychologia 12:323-330

37. Brickenkamp R 1994 Test d2. Aufmerksamkeits-Belastungs-Test 8:Auflage. Hogrefe, Göttingen, pp 28-39

38. Burgard P, Bremer HJ, Buhrdel P, Clemens PC, Monch E, Przyrembel H, Trefz FK, Ullrich K 1999 Rationale for the German recommendations for phenylalanine level control in phenylketonuria. Eur J Ped 158:46-54

39. Ullrich K, Weglage J, Oberwittler C, Pietsch M, Fünders B, van Eckhardstein H, Colombo JP 1994 Effect of L-dopa on pattern visual evoked potentials (P-100) and neuropsychological tests in untreated adult patients with phenylketonuria. J Inherit Metab Dis 17:349-352

40. Pietz J, Kreis R, Rupp A, Mayatepek E, Rating D, Boesch C, Bremer HJ 1999 Large neutral amino acids block phenylalanine transport into brain tissue in patients with phenylketonuria. J Clin Invest 103:1169-1178 